\title{
An Ultra-Low-Power Micro-Optoelectromechanical Tilt Sensor
}

\author{
Timothy G. Constandinou*†, Julius Georgiou*, and Charalambos Andreou* \\ ${ }^{*}$ Holistic Electronics Research Lab, ECE Department, University of Cyprus, Nicosia 1678, Cyprus \\ ${ }^{\dagger}$ Institute of Biomedical Engineering, Imperial College of Science, Tech. \& Medicine, London SW7 2AZ, UK \\ Email: t.constandinou@\{ucy.ac.cy,imperial.ac.uk\}, julio@ucy.ac.cy, andreouc@minatec.inpg.fr
}

\begin{abstract}
This paper presents a novel hybrid CMOS/MEMS tilt sensor with a $5^{\circ}$ resolution over a $330^{\circ}$ range. The device uses a MEMS-based semicircular mass suspended from a rigid body, projecting a shadow onto the CMOS-based optical sensor surface. A one-dimensional photodiode array arranged as a uniformly segmented ring is then used to determine the tilt angle by detecting the position of the semicircular mass. The complete sensor occupies an area of under $2.5 \mathrm{~mm} \times 2.5 \mathrm{~mm}$.

Index Terms-Tilt Sensor, Inclinometer, Vision Chip, MEMS, Microsystem
\end{abstract}

\section{INTRODUCTION}

An increasing number of medical devices including implantable prosthetics and body worn instrumentation are incorporating sense systems within and around the body. Physical constraints demand such systems to be compact and lightweight, and the need for autonomy imposes stringent power budgets on such systems. One such sensor is the inclinometer (or tilt sensor), which senses its orientation with respect to gravity.

Sensing tilt, in personalised medicine is becoming increasingly important, especially when combined with other sensor modalities. For example, a tilt sensor incorporated within a heart monitor can provide a metric on the patients activity, providing additional insights for interpreting the electrocardiogram data. In other biomedical applications, realtime, accurate tilt data can be used in therapy, for example, in correcting vestibular balance disorders.

The vast majority of tilt sensors developed to date are capacitive (or conductive) in nature, often with electrolytic fluid-filled cavities. Such systems have been developed by Bretterklieber et al. [1] and more using recently using MEMS technology [2], [3]. Zhao et al. [4] have developed a digital MEMS-based tilt sensor offering the possibility of simplifying the interface electronics. It has also been reported possible to implement inclinometers using modified accelerometers [5], [6] but such systems are typically limited in range and/or sensitivity. Other approaches include a MEMS convectionbased [7] and optical microfilter-based [8] tilt sensors.

In this paper, we present a novel ultra-low-power tilt sensor generating a digital output. This has been implemented as part of a hybrid two chip solution (MEMS microstructure/CMOS vision chip) and has been fabricated. Section II presents the system architecture and details the implementation of integrated circuit. Section III presents the simulated results,

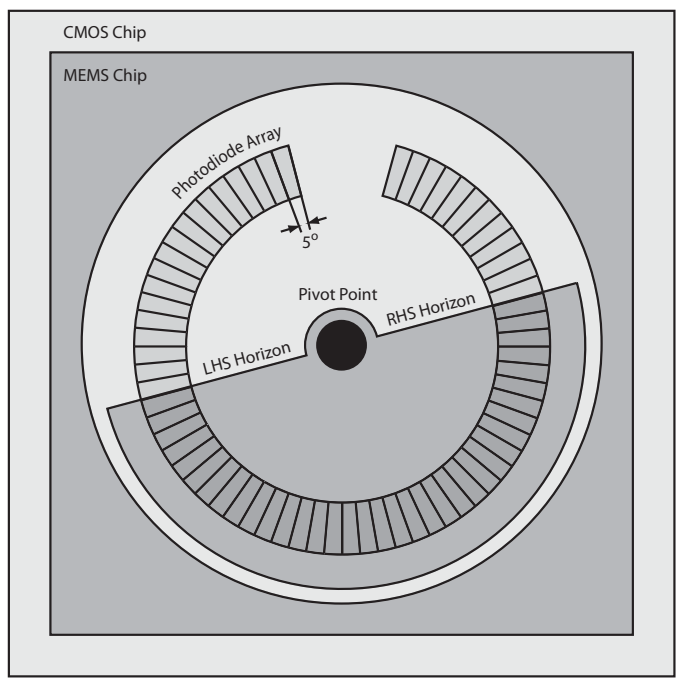

Fig. 1. The Micro-Optoelectromechanical Tilt Sensor concept, based on a two-tier stack comprising of MEMS structure on top of CMOS die. The optical detector has 66 photodetector elements arranged in $5^{\circ}$ segments to cover a $330^{\circ}$ Field-of-View (FOV).

Section IV discusses incorporation of a light emitter and Section V draws the conclusion, outlining the target system specifications.

\section{System Architecture And Circuit IMPLEMENTATION}

This tilt sensor uses a micro-mechanical semicircular mass to express the gravitational field optically and then custom CMOS vision chip to detect and resolve the vector. This concept is illustrated in Fig. 1.

The design splits the $330^{\circ}$ field of view into two $165^{\circ}$ half-planes, which we have chosen to refer to as left- and right-hand-side (LHS, RHS) horizons. This is illustrated in the top-level system architecture, shown in Fig. 2. As the micromechanical semicircular mass moves, it forms a shadow that is projected onto the surface of the CMOS chip, the edge of which crosses both the LHS and RHS horizons. Therefore, each horizon detector is required to sense the position (or tilt angle) of these edges. By taking the difference the output can encode the tilt angle using a signed, digital representation. 

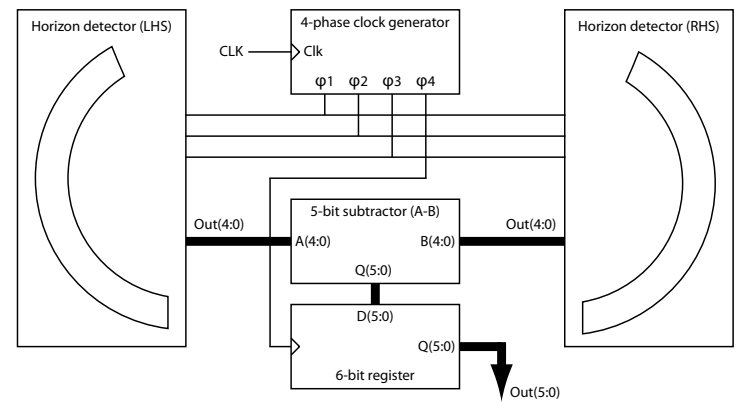

Fig. 2. The top-level system architecture comprising of two half-plane horizon detectors, clock phase generator and 2's compliment subtraction circuit.

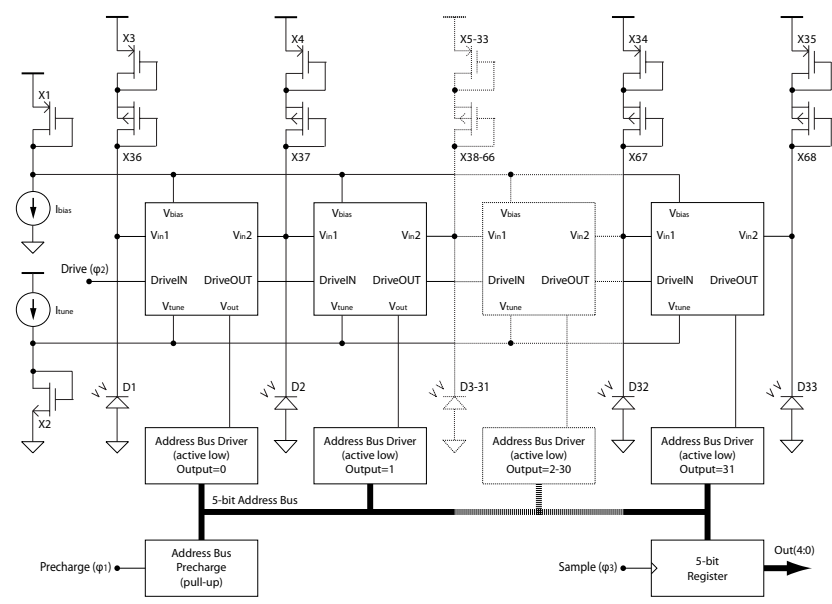

Fig. 3. Circuit schematic for the half-plane horizon detector. Each side of the tilt detector uses this horizon detector- comprising of 33 photodetector elements, and 32 edge detector cells- sequentially interrogated until the horizon (i.e. edge) is detected. The "live" element's address is then asserted onto a pre-charged bus to encode a digital position output.

\section{A. Horizon Detector Circuit}

The circuit implementation of the half-plane horizon detectors are shown in Fig. 3. This consists of 33 photodiode elements positioned equidistantly around an arc to detect the position of the semicircular mass. Between each two adjacent photodiodes, we use a discrete edge detector element (that is in total, 32 edge detectors per horizon) to compare photocurrent magnitudes and if sufficiently different flag an edge-detect signal. The output is captured using a sequence of operations. Firstly the address bus is initialised using pull-up devices to pre-charge the parasitic capacitance. Next, a DRIVE signal is propagated through the one-dimensional array, i.e. $1 \times 32$ elements, until an edge signal is encountered. On detecting an edge condition, the current position is asserted onto the address bus via the address encoder, constituting (for each element) the relevant pull-down switches. Subsequently, the DRIVE signal is terminated and the address bus is sampled onto a 5-bit register.

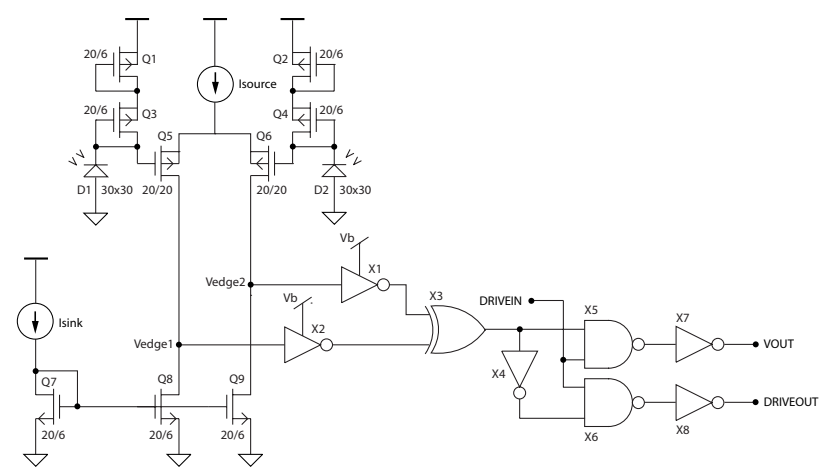

Fig. 4. Circuit schematic for the edge detector element (including photodiodes and load devices (Q1-Q4) for clarity).

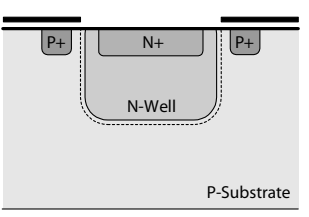

(a)

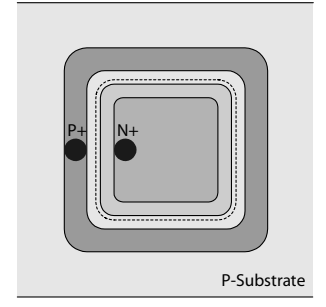

(b)
Fig. 5. Structure of n-well $/ \mathrm{p}$-substrate photodiode showing (a) side, and (b) plan view (not shown to scale or profile).

\section{B. Edge Detector Circuit}

Fig. 4 shows the circuit schematic of the edge detector element. This has been adapted from [9], [10] to the expected contrast ratio and photocurrent levels. A global tuning mechanism is maintained by driving the $I_{\text {bias }}$ and $I_{\text {tune }}$ references off-chip and internally duplicating and distributing to the individual edge detector elements. The DRIVEIN and DRIVEOUT are used to test an individual edge detectors state before propagating to test the next element. Additionally, an artificial propagation delay is implemented within the output buffer (X8 in Fig. 4) to guarantee robustness.

\section{Photodiode Design}

The photodiodes are implemented using an n-well/psubstrate junction as illustrated in Fig. 5. The different photodiode elements (66 devices) have been individually custom designed (to comply with design rule geometry) to fit within a $550 \mu \mathrm{m}$ to $600 \mu \mathrm{m}$ radius arc in $5^{\circ}$ segments. The active sensor surface for each photodiode element comes to: $4245 \mu \mathrm{m}$. This scheme can be seen in the tilt sensor core layout given in Fig. 7.

\section{4-phase Clock Generation Circuit}

The operation of the tilt sensor is centrally regulated via a 4-phase clock sequence, generated using the circuit shown in Fig. 6. This implements a looping 4-element state machine to generate the required control signals. Phase actions are: $\phi 1$ to pre-charge the bus, $\phi 2$ to interrogate the LHS and RHS horizons and drive the bus, $\phi 3$ to sample the bus result 


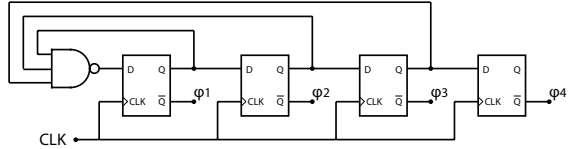

Fig. 6. Circuit schematic for the 4-phase clock generation used to sequence actions required to sense the tilt angle.

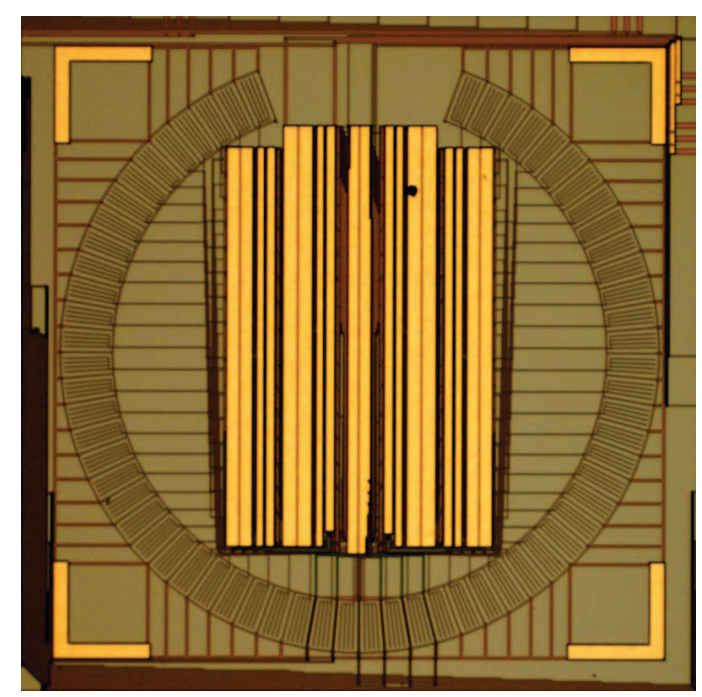

Fig. 7. Microphotograph of the tilt sensor electronics implemented in AMS $0.35 \mu \mathrm{m} 2 \mathrm{P} 4 \mathrm{M}$ CMOS technology. The dimensions are: $1250 \mu \mathrm{m} \times 1250 \mu \mathrm{m}$ (for core), and $2.5 \mathrm{~mm} \times 2.5 \mathrm{~mm}$ die dimensions, i.e. including padring and bondpads (not shown).

on horizon register, and $\phi 4$ to sample the difference (i.e. subtraction) on the output register.

\section{E. CMOS/MEMS Integrated Circuits}

Figs. 7 and 8 show the CMOS microphotograph and MEMS layout respectively.

The MEMS component has been designed such that the pivot/anchor used to suspend the mass has sufficiently small surface area in contact and good surface smoothness such that to ensure atomic forces and friction are insufficient to cause sticking. Furthermore, the mass includes anti-sticking anti-sticking holes to ensure release, based on technical data provided from Tronics foundry. During fabrication, the design incorporates a number of struts to secure the mass during processing and release. After manufacture, these struts have been intentionally designed to be breakable, thus releasing the semicircular mass into operation.

\section{Simulation Results}

The circuit was simulated using the Cadence Spectre (5.1.41isr1) simulator with foundry supplied BSIM3v3 models.

Parametric operating point simulations have been performed to determine the onset of edge detection for various bias conditions. These results are illustrated in Fig. 9. The family of curves (for different values of $I_{\text {bias }}$ ) illustrate the minimum

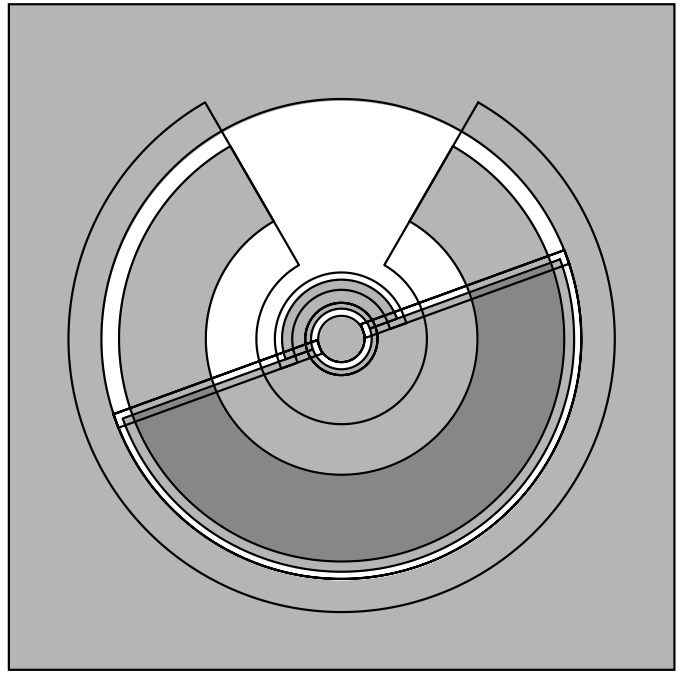

Fig. 8. MEMS layout of the micro-mechanical section of the tilt sensor implemented in the Tronics SOI/HARM technology. The dimensions are: $2.0 \mathrm{~mm} \times 2.0 \mathrm{~mm}$.

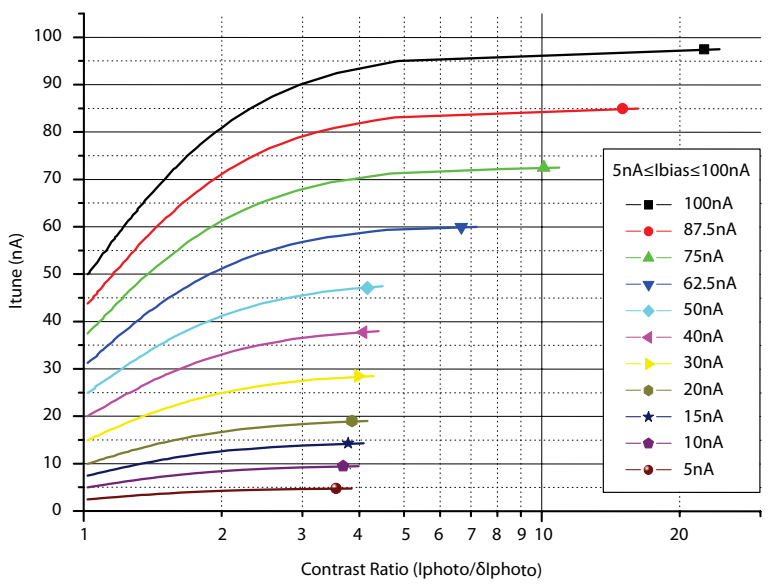

Fig. 9. Operational range (for the onset of edge detection) for the edge detector core for various bias conditions (assuming $I_{\text {photo } n}=25 \mathrm{nA}$ ).

contrast ratio $\left(I_{\text {photo }} / \delta I_{\text {photo }}\right)$ required to flag an edge detection condition. The tunability (for different values of $I_{\text {tune }}$ ) is represented in the y-axis variation. These results show to main trends of operation: (i) for $I_{\text {bias }}<I_{\text {photo }}$ the tunable range is not significantly effected by bias current level, and (ii) for $I_{\text {bias }}>I_{\text {photo }}$, increasing the bias current level significantly increases the tunable range (in particular extending the contrast ratio). It therefore envisaged that if the non-uniformity of incident light in addition to any component mismatch due to technology variation is maintained to be within the tunable limits, then relatively low bias current levels can be used (i.e. $I_{\text {bias }}=5$ to $10 \mathrm{nA}$.

A top-level transient simulation illustrates the system-level operation of the tilt sensor, presented in Fig. 10. This simulation assumes an average photocurrent $I_{\text {photo }}$ of $25 \mathrm{nA}$, an ambient off-current of $1 \mathrm{nA}$, and uses a system clock of $500 \mathrm{KHz}$. The total power consumption is $33 \mu \mathrm{W}$. 


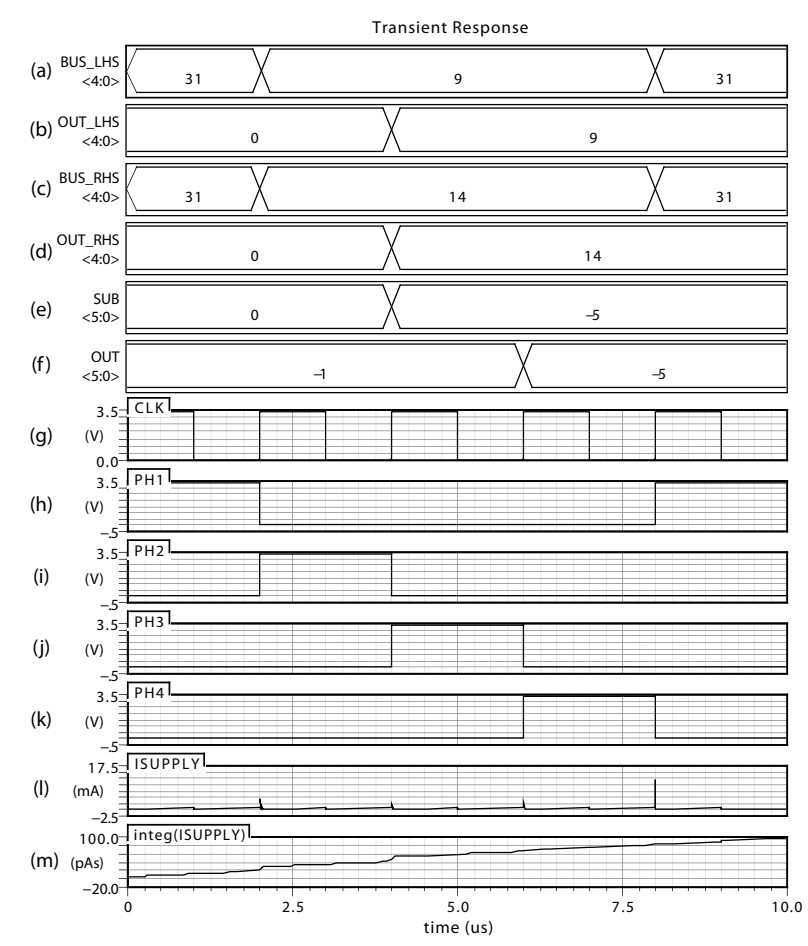

Fig. 10. Top level transient simulation for a tilt angle of $-15^{\circ}$. Results shown are: (a) LHS Horizon Bus (5-bit), (b) LHS Horizon Output (5-bit), (c) RHS Horizon Bus (5-bit), (d) RHS Horizon Output (5-bit), (e) Subtraction Result (6-bit), (f) Final Output (6-bit), (g) Clock, (h-k) $\phi 1-\phi 4$, (l) Current Supply, and (m) Integral of Current supply.

The functioning of the system can easily be traced by following the transient simulation results shown in Fig. 10 as follows: (i) On the rising edge of $\phi 1$, the two buses are precharged (result=31). (ii) On the rising edge of $\phi 2$, the horizon detector results are asserted onto the buses (results of 9 and 14 for LHS and RHS respectively). (iii) On the rising edge of $\phi 3$ the values on the buses are sampled to the horizon output registers and finally, (iv) on the rising edge of $\phi 4$, the subtracted result (from asynchronous adder) is sampled on the output register.

\section{Discussion}

One aspect of the sensor that has been omitted from the scope of this letter is the light source. To ensure robustness in dark and fluctuating light conditions, the sensor is envisaged to incorporate a dedicated light-source. This can be a small area $\left(2 \mathrm{~mm}^{2}\right)$, focused (i.e. narrow viewing area) lightemitting diode (LED) to stimulate the photodetectors within the vision chip. The composite emitter/sensor can then be packaged (eg. as in optoisolators) to seal the components from environmental exposure. To minimise power consumption, the light source can be biased at low intensity and/or pulsed, as the photodetectors cover a large sense area and will therefore be extremely efficient. The minimum light intensity (and thus power consumption) is limited by: (i) the photodiode dark current, (ii) the minimum detectable contrast ratio (for robust edge detection), and (iii) the required sensory response time.

\begin{tabular}{ll}
\hline CMOS Technology & AMS $0.35 \mu \mathrm{m}$ 2P4M CMOS \\
MEMS Technology & Tronics SOI/HARM \\
Supply Voltage & $3.3 \mathrm{~V}$ \\
Circuit Size & $2500 \mu \mathrm{m} \times 2500 \mu \mathrm{m}$ \\
Device Count & 4312 \\
Input Light Intensity (range) & $10 \mathrm{nW} / \mathrm{mm}^{2}$ to $100 \mu \mathrm{W} / \mathrm{mm}^{2}$ \\
Input System Clock (range) & $100 \mathrm{~Hz}$ to $1 \mathrm{MHz}$ \\
Input Current Bias (range) & $1 \mathrm{nA}$ to $50 \mathrm{nA}$ \\
Tilt Angle Range & $330^{\circ}\left( \pm 165^{\circ}\right)$ \\
Tilt Resolution & $5^{\circ}$ \\
Effective Dynamic Range & $36 \mathrm{~dB}(6 \mathrm{bits})$ \\
System Power Consumption & $\dagger 10-35 \mu \mathrm{W}$ \\
& \\
\hline
\end{tabular}

†excluding light emitter consumption

TABLE I

TARGET DESIGN SPECIFICATIONS

\section{CONCLUSION}

In this paper we have presented a hybrid CMOS/MEMS inclinometer that uses a photodetector array to detect the position of a suspended microsystem. With a uniform array of radially positioned photodiodes, the tilt sensor achieves a $5^{\circ}$ resolution over a $330^{\circ}$ range. The system power consumption depends highly on the incident light intensity but expected to be within the range $10-35 \mu \mathrm{W}$.

The target system specifications are given in Table I.

\section{ACKNOWLEDGMENT}

This work was supported by the Cyprus Research Promotion Foundation (RPF), grant no.: $\Pi \Delta E-0505 / 07$.

\section{REFERENCES}

[1] T. Bretterklieber, H. Zangl, and G. Brasseur, "Impacts on the accuracy of a capacitive inclination sensor," Proc. IEEE Instrumentation and Measurement Technology Conference, vol. 3, pp. 2315- 2319, 2004.

[2] R. A. Yotter, R. R. Baxter, S. Ohno, S. D. Hawley, and D. M. Wilson, "On a Micromachined Fluidic Inclinometer," Proceedings of IEEE International Conference on Transducers, Solid-State Sensors, Actuators and Microsystems, vol. 2, pp. 1279-1282, 2003.

[3] K.-S. Kang, H. Jung, D.-S. Kim, B.-J. Kwon, C. J. Kim W.-J. Kim, S.-Y. Choi, J.-H. Lee, J.-K. Shin, and S. H. Kong, "Design and fabrication of a MEMS-based electrolytic tilt sensor," Proceedings of the IEEE Conference on Microprocesses and Nanotechnology, pp. 216-217, 2005.

[4] L. Zhao, and E. Yeatman, "Micro Capacitive Tilt Sensor for Human Body Movement Detection," Proceedings of Body Sensor Networks, vol. 39, pp. 195-200, 2007.

[5] D. Lapadatu, S. Habibi, B. Reppen, G. Salomonsen, and T. Kvisteroy, "Dual-axes capacitive inclinometer/low-g accelerometer for automotive applications," IEEE International Conference on Micro Electro Mechanical Systems, vol. 39, pp. 34-37, 2001.

[6] S. Luczak, et al., "Sensing Tilt With MEMS Accelerometers," IEEE Sensors Journal, vol. 6, no. 6, pp. 1669-1675, 2006

[7] S. Billat, H. Glosch, M. Kunze, F. Hedrich, J. Frech, J. Auber, and W. Lang, "Convection-based Micromachined Inclinometer Using SOI Technology," Proceedings of IEEE International Conference on Micro Electro Mechanical Systems, pp. 159-161, 2001.

[8] N. C. Tien, "Micro-optical Inertial Sensors using Silicon MEMS," Proceedings of IEEE Aerospace Conference, vol. 1, pp. 437-443, 1998.

[9] T. G. Constandinou, J. Georgiou, and C. Toumazou, "Nano-power mixed-signal tunable edge-detection circuit for pixel-level processing in next generation vision systems," IET Electronics Letters, vol. 39, no. 25, pp. 1774-1776, 2003.

[10] T. G. Constandinou and C. Toumazou, "A micropower centroiding vision processor," IEEE Journal of Solid-State Circuits, vol. 41, no. 6, pp. 1430-1443, 2006. 